\title{
A Relaxation Method for the Determination of Moderately Rapid Reaction Rates near Chemical Equilibrium
}

\author{
STIG L J U NGGREN and OLE LAM M
}

The Royal Institute of Technology, Division of Physical Chemistry, Stockholm 70, Sweden

\begin{abstract}
A new relaxation method, based on the equilibrium displacement produced by a rapid pressure rise, is described. Using the present technique, the pressure was raised from 1 to 150 atmospheres in about one twentieth of a second. The relaxation to equilibrium was followed by a continuous registration of the conductivity of the sample solution. This technique was employed to study the kinetics near equilibrium of the carbon dioxide-water system. It was also found possible to calculate the true dissociation constant of carbonic acid.
\end{abstract}

\section{SYMBOLS}

$b_{r s}=$ constants defined in text

$C_{p}=$ specific heat

$C=$ total concentration

$c^{\circ}=$ equilibrium concentration

$c_{\mathrm{o}}=$ concentration of undissociated constituent

$c_{+}=$concentration of positive ion

$c_{-}=$concentration of negative ion

$\bar{H}^{\circ}=$ partial molar enthalpy of standard state (referred to infinite dilution)

$J=$ cell constant

$K=$ equilibrium constant in general

$K_{s}=$ true dissociation constant of $\mathrm{CO}_{2}$

$K_{h}=$ hydration constant of $\mathrm{CO}_{2}$

$K_{s h}=$ apparent dissociation constant of $\mathrm{CO}_{2}$

$k_{i}=$ forward rate constant

$k_{r}=$ reverse rate constant

$l=$ eqivalent ionic conductance

$p=$ pressure; algebraic expression defined in the text

$q=$ algebraic expression defined in the text

$R=$ gas constant; cell resistance

$S=$ entropy

$T=$ absolute temperature 


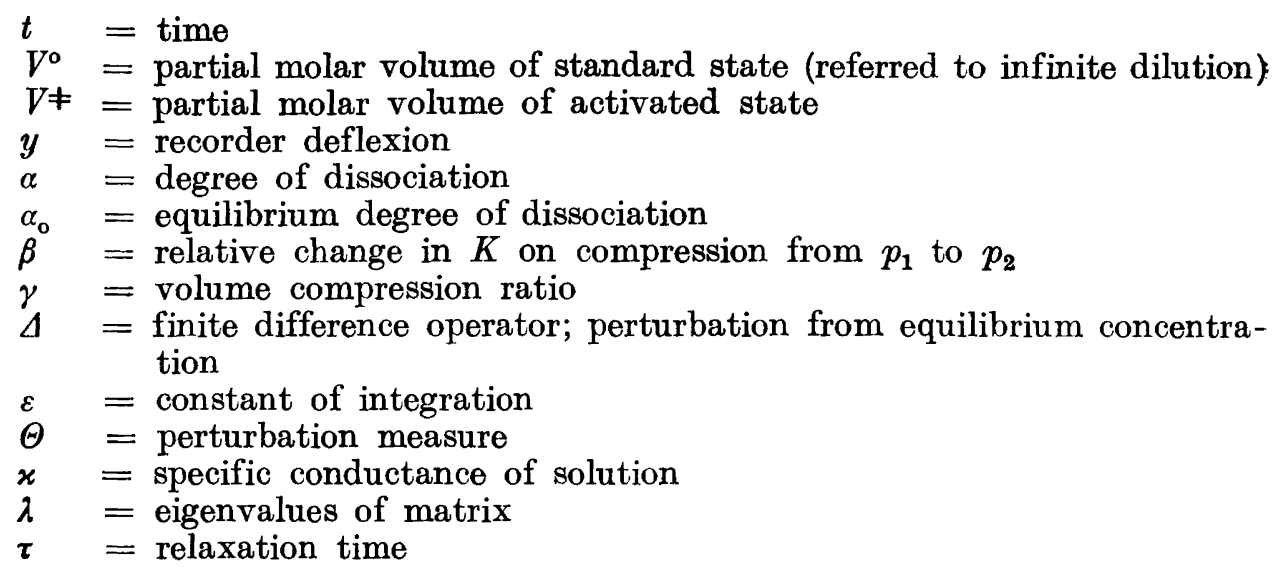

\section{INTRODUCTION}

In connection with other investigations at this Institute there arose a need for a method suitable for the study of the kinetics of certain reactions with half-times ranging from $0.05 \mathrm{sec}$ up to a few minutes. All reactions which were studied involved ionic species. For this reason it seemed most convenient to utilize the conductivity of the solutions. Of the existing methods $^{1}$ for studying fast reactions, only the rapid flow method of Hartridge and Roughton ${ }^{2}$ seemed to be applicable in the region of rates mentioned. An estimation showed, however, that it would be difficult to adapt the flow method successfully to conductivity registration without sacrificing the low liquid consumption which is characteristic of certain modifications of this method. From the point of view of general experimental simplicity, a relaxation method seemed most attractive. This led the authors to the development of what might be called a pressure jump method. By recording the variation of conductivity following the application of a rapid pressure rise, the rate at which the new equilibrium was approached could be studied. There is a simple relation between the relaxation time (time lag) and the reaction rates (cf. Refs. $\left.{ }^{3,4}\right)$. A rather similar method, the temperature jump method, has been indicated by Eigen ${ }^{5}$.

A still simpler relaxation method, which to the authors' knowledge, has not been tried as yet, would be a rapid dilution of the sample solution followed by, for example, conductivity registration.

The pressure jump method is not confined to solutions having an electric conductivity. The variation of colour, temperature or dielectric constant of the solution may be used as well. In addition to the relaxation process, the pressure dependence of the equilibrium constant can be studied by the same experimental arrangement.

The original flow method ${ }^{2},{ }^{2}$ requires large quantities of liquid. In later modifications ${ }^{2}$, e. g. the stopped flow and accelerated flow methods, this drawback has been overcome. The flow method has hitherto been applied mainly to biological systems, e. g. enzyme reactions. The rate of hydration and dehydration of carbon dioxide and the rate of the conversion $\mathrm{Cr}_{2} \mathrm{O}_{7}^{2-}-\mathrm{HCrO}_{4}^{-}$have, however, also been determined in this way'.

Acta Chem. Scand. 12 (1958) No. 9 
Relaxation methods ${ }^{1}$ have attracted much attention in recent years. In addition to the pressure jump method described in this paper and the remperature jump method already mentioned, we have, for example, the method utilizing the equilibrium displacement caused by a strong electric field, the so called dissociation field effect (or second Wien effect) ${ }^{1,3}$. Owing to the enormous production of Joule heat per unit time, this method can only be used for very fast reactions. The same thing is true for the sound absorption method ${ }^{1}$.

Proton magnetic resonance has been applied, for example, to the study of the extremely rapid protolysis of ammonia and various amines $\mathbf{1 , 7}$.

A dielectric dispersion method has recently been used to study the dissociation rate of boric acid ${ }^{\mathbf{8}}$.

Polarographic and related methods ${ }^{9-14}$ are applicable in cases where the electrode process is preceded by the reaction to be studied. As an example of a successful application of this technique, the determination of the dissociation rate of the complex ion $\left[\mathrm{Cd}(\mathrm{CN})_{4}\right]^{2-}$ may be mentioned ${ }^{15}$.

\section{BASIC THEORY}

The effects of pressure on the conductivity of an electrolyte are twofold. First, the ionic mobilities are changed, due in the first place to the change in viscosity of the solvent. Second, there is a displacement of the equilibrium position (i.e. a change in the equilibrium degree of dissociation) which we utilize to study the rate of approach to the new equilibrium at the higher pressure. The equilibrium perturbation is to be ascribed partly to the change in the equilibrium constant and partly to the pure volume effect, i.e. the increase of the concentrations due to the volume compression.

1. The pressure effect on the equilibrium constant

For the variation of the equilibrium constant, $K$, with pressure, $p$, under adiabatic conditions we have

$$
(\partial \ln K / \partial p)_{S}=(\partial \ln K / \partial p)_{T}+(\partial \ln K / \partial T)_{p} \quad(\partial T / \partial p)_{S},
$$

where $T$ is the absolute temperature and $S$ the entropy of the solution. Now, from thermodynamics we know that

$$
\begin{gathered}
(\partial \ln K / \partial p)_{T \mathfrak{p}}=-\Delta V^{\circ} / R T \\
(\partial \ln K / \partial T)_{p}=\Delta H^{\circ} / R T^{2} \\
(\partial T / \partial p)_{S}=T(\partial V / \partial T)_{p} / C_{p}
\end{gathered}
$$

where $V^{\circ}$ and $H^{\circ}$ are the volume and enthalpy of the standard states (referred to infinite dilution).

Realizing that

$$
(\partial \ln K / \partial p)_{s}=\frac{1}{R T} \cdot\left[-\Delta V^{\circ}+\Delta H^{\circ}(\partial V / \partial T)_{p} / C_{p}\right]
$$

$$
\ln \left(K_{2} / K_{1}\right)=\int_{p_{1}}^{p_{2}}(\partial \ln K / \partial p)_{S} \mathrm{~d} p
$$


and introducing the ratio

we have

$$
\beta=K_{2} / K_{1}
$$

$$
\begin{gathered}
\beta=\exp \left[\int_{p_{1}}^{p_{2}}(\partial \ln K / \partial p)_{s} \mathrm{~d} p\right]= \\
=\exp \left[\int_{p_{1}}^{p_{1}} \frac{-\Delta V^{\circ}+\Delta H^{\circ}(\partial V / \partial T)_{p} / C_{p}}{R T} \cdot \mathrm{d} p\right]
\end{gathered}
$$

When working with dilute aqueous solutions, we may disregard the second term within the bracket in the expression for $(\partial \ln K / \partial p)_{S}$ since $(\partial V / \partial T)_{p}$ is very small for water at room temperature (and zero at the temperature of maximum density). If the equilibrium involves ionic species, the change in $K$ is accompanied by a corresponding change in the conductivity of the solution.

As to the magnitude of the pressure effect, we find that if $\Delta V^{\circ}=1 \mathrm{~cm}^{3}$ per mole, then a change of $p$ from 1 to 100 atm will produce a change in $K$ of $4 \times 10^{-3}$. This is sufficient to permit registration of the relaxation process.

\section{The volume effect}

The volume effect is of course much smaller in a solution than in a gas mixture, but it is comparable in magnitude to the change in $K(i . e . \beta)$ given above. As an example let us study the dissociation equilibrium

$$
\begin{aligned}
& \mathrm{AB} \rightleftharpoons \mathrm{A}^{+}+\mathrm{B}^{-} \\
& c_{\mathrm{o}} c_{+} \quad c_{-}
\end{aligned}
$$

According to the law of mass action we have

$$
c_{+} c_{-} / c_{\mathrm{o}}=K
$$

If, now, the pressure is rapidly raised, say, from 1 to $100 \mathrm{~atm}$, all concentrations are instantaneously increased in the same ratio, $\gamma$, as the volume decrease of the solution.

$$
\begin{aligned}
c_{\mathrm{o}}^{\prime} & =\gamma c_{\mathrm{o}} \\
c_{+}^{\prime} & =\gamma c_{+} \\
c_{-}^{\prime} & =\gamma c_{-}
\end{aligned}
$$

$\gamma$ can be calculated if the compressibility of the solution is known. As $c$ changes to $c^{\prime}, K$ will simultaneously change to $K^{\prime}$

$$
K^{\prime}=\beta K
$$

where $\beta$ can be calculated using the equation deduced in the foregoing section. Acta Chem. Scand. 12 (1958) No. 9 
The equilibrium is perturbed as a result of the changes in $c$ and $K$. As a measure for the perturbation, we introduce a quantity $\Theta$, defined by the relation

Thus

$$
c_{+}^{\prime} c_{-}^{\prime} / c_{0}^{\prime}=(1+\Theta) K^{\prime}
$$

i.e.

$$
\gamma c_{+} \gamma c_{-} / \gamma c_{\mathrm{o}}=(1+\Theta) \beta K
$$

$$
\Theta=(\gamma-\beta) / \beta
$$

Obviously, the two effects, $\gamma$ and $\beta$, may occasionally cancel. On the other hand, it is possible to obtain a measurable effect even in such cases where $\beta$ is very small or equal to zero. It is hard to make any predictions since $\Delta V^{\circ}$ varies appreciably from case to case. (cf. Ref. $\left.{ }^{16}\right)$.

3. The change in the ionic mobilities

As discussed by Bridgman ${ }^{17}$, the ionic mobilities are themselves functions of the pressure. However, this effect, being instantaneous, has no influence on the relaxation time.

4. The relaxation

In order to get an idea of the relaxation process we will study the case of a weak binary electrolyte ${ }^{*}$ which dissociates according to the formula

Then

$$
\begin{array}{rrr}
\mathrm{AB} \underset{k_{r}}{\stackrel{k_{r}}{\rightleftharpoons}} & \mathrm{A}^{+}+\mathrm{B}^{-} \\
C(1-\alpha) & C \alpha \quad C \alpha
\end{array}
$$

$$
\frac{C \alpha_{\mathrm{o}}^{2}}{1-\alpha_{\mathrm{o}}}=K
$$

where $\alpha_{\mathrm{o}}$ is the equilibrium degree of dissociation and $\alpha$ the actual degree of dissociation. $k_{f}$ and $k_{r}$ are the forward and reverse rate constants respectively. The rate equation of the system is

$$
\frac{\mathrm{d} \alpha}{\mathrm{d} t}=k_{f}\left(1-\alpha-\frac{C \alpha^{2}}{K}\right)
$$

On integration (cf. Ref. ${ }^{4}$ ) at constant $K$ and $k_{\mathrm{f}}$ we obtain

$$
\frac{\alpha-\alpha_{\mathrm{o}}}{\alpha+\frac{\alpha_{\mathrm{o}}}{1-\alpha_{\mathrm{o}}}}=\varepsilon \mathrm{e}^{-t / \tau}
$$

* An example of a more complicated case will be encountered below in connection with the system carbon dioxide-water.

Acta Chem. Scand. 12 (1958) No. 9 
where is a constant and

$$
\tau=\frac{1-\alpha_{\mathrm{o}}}{k_{r} C\left(2-\alpha_{\mathrm{o}}\right) \alpha_{\mathrm{o}}}
$$

$\tau$ is the so-called Langevin time lag. The expression may be rewritten in the form

$$
\therefore \quad \tau=\frac{\alpha_{0}}{k_{f}\left(2-\alpha_{0}\right)}
$$

If $\alpha-\alpha_{0}=\Delta \alpha$ is small in comparison with $\alpha_{0}$, the integrated rate equation becomes approximately

$$
\Delta \alpha=(\Delta a)_{0} \mathrm{e}^{-t / \boldsymbol{\tau}_{1}}
$$

where $(\Delta \alpha)_{\mathrm{o}}$ refers to $t=0$.

The specific conductance of the relaxing solution is proportional to $\alpha$ :

$$
x=10^{-3} C \alpha\left(l_{\mathrm{A}+}+l_{\mathrm{B}-}\right)
$$

where $l_{\mathrm{A}+}$ and $l_{\mathrm{B}-}$ denote the equivalent conductances of $\mathrm{A}_{i+}^{+}$and $\mathrm{B}^{-}$respectively at the elevated pressure.

Hence

$$
\Delta x=(\Delta x)_{0} \mathrm{e}^{-t / \tau}
$$

Using this expression, $\tau$ may be determined by following the variation of $\varkappa$.

The expression for $\tau$ shows that it depends on $\alpha_{0}$. Since $\alpha_{0}$ depends in turn on $C$ and $K$, a suitable concentration range must be chosen in each particular case in order that $\tau$ may assume a value suitable for measurement.

Having determined $\tau$, the question árises as to whether the value obtained agrees with the reaction rate at atmospheric pressure. For the variation of the rate constants with pressure we have ${ }^{18}$

$$
\left(\frac{\partial \ln k}{\partial p}\right)_{T !}=\frac{\Delta V \neq}{R T}
$$

where $\Delta V \neq$ refers to the activated state. Obviously this effect is of the same order of magnitude as the change in $K$, i.e. about $1 \%$ for a pressure variation of $100 \mathrm{~atm}$. As a rule this is within the limit of errors in the determination of $\tau$ and $k$.

\section{THE APPARATUS}

A conductivity cell, two types of which are shown in Figs. 1 and $2 *$, was enclosed in a pressure vessel of stainless steel, Fig. 3 . The pressure was transmitted from the pressure medium (water or paraffin oil) to the solution in the cell by means of a flexible polythene tube, Fig. 1. This simple arrangement proved completely satisfactory in practice. When water was used as pressure

* The cell in Fig. 2 has the advantage of giving a constant current density in every part of the electrode surfaces. 


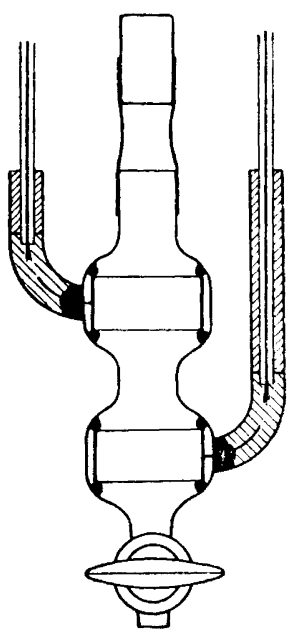

Fig. 1. Conductivity cell. The polythene tube on top of the cell transmits the pressure.
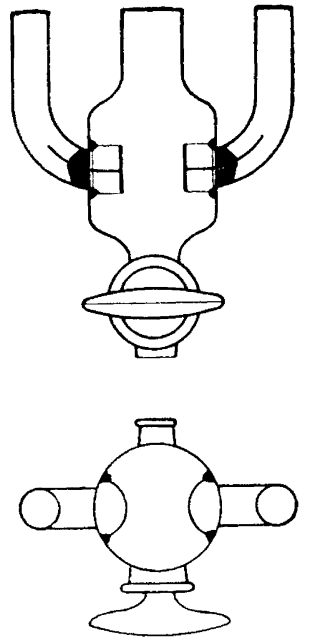

Fig. 2. Conductivity cell. The electrodes are curved (harmonic circles) so that the current density is nearly constant over the whole electrode surface.

medium, the knurled screws inside the vessel and the connections to the insulated leads were completely embedded in picein in order to avoid conduction through the water. The rapid pressure rise was achieved simply by connecting the pressure vessel to a $150 \mathrm{~atm}$ nitrogen cylinder and rapidly opening the

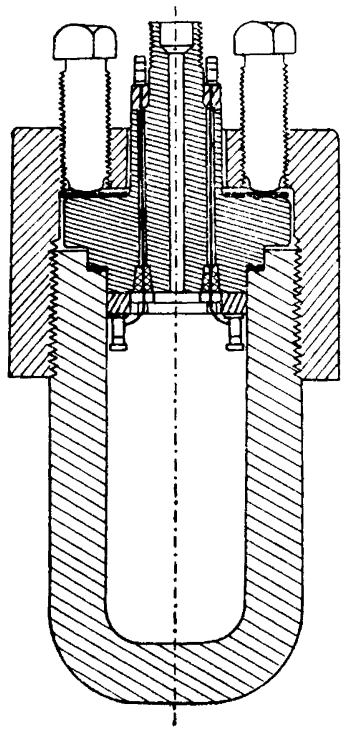

Fig. 3. Pressure vessel. The electrode leads pass through teflon cones tightening according to the "principle of the unsupported area" 17. 


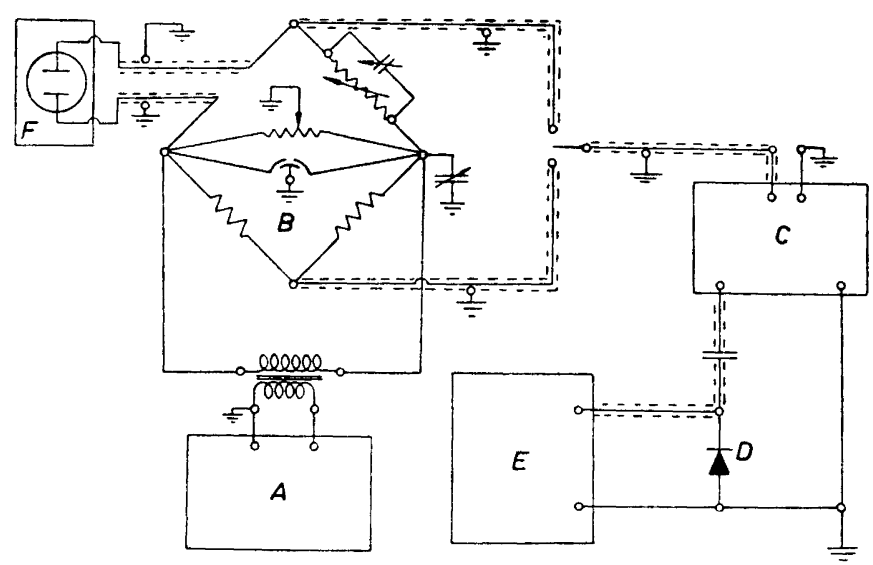

Fig. 4. Recording system. A, oscillator; B, Wheatstone bridge (separately balanced against earth); C, amplifier; D, rectifier; E, recorder; F, conductivity cell.

stopcock. In this way the pressure variation could be obtained without shock or vibration in less than one twentieth of a second. This sets the lower limit to the range of time-lags that can be determined. However, it should possible to improve the present form of the method.

The recording system is shown schematically in Fig. 4. The cell, the isolated! leads of which passed through the cover of the pressure vessel, forms one branch of a Wheatstone bridge fed from an audio frequency oscillator*. The detector signal, which at low degress of unbalance is approximately proportional to the relative variation of the resistance of the cell, was amplified ** and rectified. The DC voltage from the copper rectifier was then recorded $* * *$. The working conditions have to be chosen in such a way that, as far as possible, the recording system is linear in the whole range used.

Fig. 5 shows an actual registration with $0.01 \mathrm{M} \mathrm{KCl}$ in the cell and paraffin oil as pressure medium. In this case, the whole of the change in conductivity is due to the change in ionic mobilities and to the pure volume effect.

Fig. 6 shows a registration from an experiment with a carbon dioxide solution. The instantaneous change, $\mathrm{AB}$, is due to the same reasons as those for $\mathrm{KCl}$ and also to a displacement of the rapid equilibrium: $\mathrm{H}_{2} \mathrm{CO}_{3} \rightleftharpoons \mathrm{H}^{+}+\mathrm{HCO}_{3}^{-}$. The slow change, $\mathrm{BC}$, on the other hand, reflects the relaxation of the hydration reaction of $\mathrm{CO}_{2}$. The theory of this will be given below.

* At frequencies above 5000 cycles/sec, the mass of the recorder galvanometer prevents the superimposed AC component (ripple) from being recorded.

** In order to avoid transient distortion (from the building-up process), the band width of the amplifier must not be too small. (cf. Ref. ${ }^{18}$ a). For this reason, an untuned amplifier was used.

*** A direct writing recorder, the Mingograph, manufactured by the Elema Co., Stockholm, Sweden, was used. The ink is sprayed on to the recording paper by a small, light nozzle. This eliminates the friction between pen and paper and enables rapid processes to be recorded on ordinary paper. The instrument was originally designed for medical purposes.

Acta Chem. Scand. 12 (1958) No. 9 


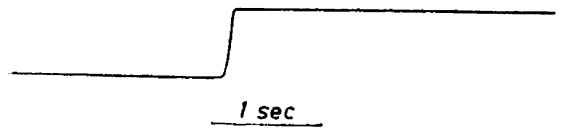

Fig. 5. Record obtained with $0.01 \mathrm{M} \mathrm{KCl}$.

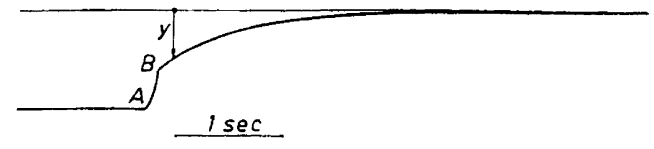

Fig. 6. Record obtained with a $\mathrm{CO}_{2}$ solution. $A B$, rapid change; $B C$, slow change (relaxation curve).

Fig. 6 also illustrates the method of evaluation used. We have

$$
y=y_{\mathrm{o}} \mathrm{e}^{-t / \tau}
$$

Hence, if $y$ is plotted in a logarithmic scale versus time, then $-1 / \tau$ is obtained as the slope of the line.

Theoretically there should be no lower limit for the rates of reactions which can be determined by the method. The following facts have, however, to be considered. On compression to a hundred atmospheres, the temperature of the medium may rise by several degrees.* Thus heat is slowly transferred to the conductivity vessel, changing the conductivity of the electrolyte. This may mask the change in conductivity if the reaction studied is slow (large $\tau)^{* *}$. If the reaction is fast the heat of the compression is less disturbing ***. However, the advantage of using a pressure medium at the temperature of its density maximum (if it has one), e.g. water at $4^{\circ} \mathrm{C}$, becomes apparent. For fast

* The adiabatic heat of compression obeys the equation already stated:

$$
(\partial T / \partial p)_{S}=\left(T / C_{p}\right)(\partial V / \partial T)_{p}
$$

Assuming that $(\partial T / \partial p)_{S}$ remains constant, we may easily calculate the temperature rise on compression to a hundred atmospheres. Approximate values for some common substances are (at room temperature):

Substance
Pentane
Ethyl ether
Benzene
Rubber
Paraffin
Glycerol
Mercury
2.5 \% KCl in aq.
Water
$\quad$ Brass
Glass
Porcelain
Nitrogen gas

Temperature rise $\left({ }^{\circ} \mathrm{C} / 100\right.$ atm $)$
3.5
3.15
2.45
0.84
0.84
0.49
0.28
0.16
0.07 .
0
0.10
0.03

** In experiments with solutions having a high conductivity, the Joule heat evolved in the eell must also be considered.

*** For such rapid reactions where the heat conduction can be neglected, the temperature jump method may have an advantage over the present one, since it permits a more rapid application of the perturbation. However, the rate of application of the pressure might be considerably increased using, for example, "a bursting membrane" technique (compare e.g. ${ }^{19,20}$ ). 
processes, paraffin oil is superior since the turbulence resulting from the application of pressure is damped much more rapidly in paraffin oil than in water. In addition, paraffin oil is a superior electrical insulator. It was found practical to use agar in the water to increase its viscosity to approximately that of paraffin oil.

The electrodes of the cell were treated with $\mathrm{HNO}_{3} / \mathrm{HCl}$ and then carefully washed before each run. In the experiments with carbon dioxide solutions, unplatinized electrodes were used in order to prevent adsorption on the electrode surfaces.

\section{THE SYSTEM CARBON DIOXIDE-WATER}

a. Relaxation time. The system carbon dioxide-water is an example of a case which is more complicated than the simple binary electrolyte case treated above. In this system, there is an intermediate stage, the hydration of $\mathrm{CO}_{2}$, so that the dissociation proceeds according to the formula

$$
\mathrm{CO}_{2}+\mathrm{H}_{2} \mathrm{O} \underset{k_{2}}{\stackrel{k_{1}}{\rightleftharpoons}} \mathrm{H}_{2} \mathrm{CO}_{3} \underset{k_{4}}{\stackrel{k_{3}}{\rightleftharpoons}} \mathrm{H}^{+}+\mathrm{HCO}_{3}^{-}
$$

There is also a competing reaction path

$$
\mathrm{CO}_{2}+\mathrm{OH}^{-} \rightleftharpoons \mathrm{HCO}_{3}^{-}
$$

which, however, does not become important unless $\mathrm{p} \mathrm{H}>8$ (cf. Ref. $\left.{ }^{21}\right)$. Hence, the system will obey the following rate equations

$$
\left\{\begin{array}{l}
\frac{\mathrm{d} c_{\mathrm{CO}_{2}}}{\mathrm{~d} t}=-k_{1} c_{\mathrm{CO}_{2}}+k_{2} c_{\mathrm{H}_{2} \mathrm{CO}_{3}} \\
\frac{\mathrm{d} c_{\mathrm{H}_{2} \mathrm{CO}_{3}}}{\mathrm{~d} t}=k_{1} c_{\mathrm{CO}_{3}}-k_{2} c_{\mathrm{H}_{2} \mathrm{CO}_{3}}-k_{3} c_{\mathrm{H}_{2} \mathrm{CO}_{3}}+k_{4} c_{ \pm}^{2} \\
\frac{\mathrm{d} c_{ \pm}}{\mathrm{d} t}=k_{3} c_{\mathrm{H}_{2} \mathrm{CO}_{3}}-k_{4} c_{ \pm}^{2}
\end{array}\right.
$$

where

We will now introduce

$$
c_{ \pm}=c_{\mathrm{H}+}=c_{\mathrm{HCO}}
$$

$$
\left\{\begin{array}{l}
c_{\mathrm{CO}_{2}}=c_{\mathrm{CO}_{2}}^{\circ}+\Delta_{1} \\
c_{\mathrm{H}_{2} \mathrm{CO}_{\mathrm{s}}}=c_{\mathrm{H}_{2} \mathrm{C}_{3} \mathrm{O}}^{\circ}+\Delta_{2} \\
c_{ \pm}=c_{ \pm}^{\circ}+\Delta_{3}
\end{array}\right.
$$

where $\Delta_{1}, \Delta_{2}$, and $\Delta_{3}$ denote small deviations from the equilibrium concentrations (denoted $c^{\circ}$ ).

$$
\left\{\begin{array}{l}
0=k_{1} c_{\mathrm{CO}_{3}}^{\circ}+k_{2} c_{\mathrm{H}_{2} \mathrm{CO}_{3}}^{\circ} \\
0=k_{1} c_{\mathrm{CO}_{2}}^{\circ}-k_{2} c_{\mathrm{H}_{2} \mathrm{CO}_{3}}^{\circ}-k_{3} c_{\mathrm{H}_{2} \mathrm{CO}_{3}}^{\circ}+k_{4}\left(c_{ \pm}^{\circ}\right)^{2} \\
0=k_{3} c_{\mathrm{H}_{2} \mathrm{CO}_{3}}^{\circ}-k_{4}\left(c_{ \pm}^{\circ}\right)^{2}
\end{array}\right.
$$

Acta Chem. Scand. 12 (1958) No. 9 
For small $\Delta$ we may put

$$
c_{ \pm}^{2} \approx\left(c_{ \pm}^{\circ}\right)^{2}+2 c_{ \pm}^{\circ} \Delta_{3}=\left(c_{ \pm}^{\circ}\right)^{2}+k_{5} \Delta_{3} / k_{4}
$$

where $k_{5}=2 c_{ \pm}^{\circ} k_{4}$

In this case there results

$$
\left\{\begin{array}{l}
\frac{\mathrm{d} \Delta_{1}}{\mathrm{~d} t}=-k_{1} \Delta_{1}+k_{2} \Delta_{2} \\
\frac{\mathrm{d} \Delta_{2}}{\mathrm{~d} t}=k_{1} \Delta_{1}-k_{2} \Delta_{2}-k_{3} \Delta_{2}+k_{5} \Delta_{3} \\
\frac{\mathrm{d} \Delta_{3}}{\mathrm{~d} t}=k_{3} \Delta_{2}-k_{5} \Delta_{3}
\end{array}\right.
$$

or written in matrix notation

$$
\frac{\mathrm{d} \Delta}{\mathrm{d} t}+A \Delta=0
$$

where

$$
A=\left(\begin{array}{ccc}
k_{1} & -k_{2} & 0 \\
-k_{1} & k_{2}+k_{5} & -k_{5} \\
0 & -k_{3} & k_{5}
\end{array}\right) ; \quad \Delta=\left(\begin{array}{c}
\Delta_{1} \\
\Delta_{2} \\
\Delta_{3}
\end{array}\right)
$$

The general solution of this system, which is easily obtained on diagonalization of the matrix (cf. e.g. Frost and Pearson ${ }^{22}$ or Matsen and Franklin ${ }^{23}$ ), can be written as linear combinations of exponential functions

$$
\Delta=e^{-A t} \Delta_{0} \quad \text { or } \quad \Delta_{r}=\sum_{s=1}^{3} b_{r s} e^{-\lambda_{s}}
$$

where $b_{r s}$ are constants (components of the eigenvectors) and $\lambda_{s}$ are the eigenvalues of the matrix, or solutions of the secular equation:

$$
\left|\begin{array}{ccc}
k_{1}-\lambda & -k_{2} & 0 \\
-k_{1} & k_{2}+k_{3}-\lambda & -k_{5} \\
0 & -k_{3} & k_{5}-\lambda
\end{array}\right|=0
$$

This equation has three solutions

$$
\begin{aligned}
& \lambda_{1}=0 \\
& \lambda_{2}=\frac{1}{2}(p+q) \\
& \lambda_{3}=\frac{1}{2}(p-q)
\end{aligned}
$$


where

$$
\begin{aligned}
& p=k_{1}+k_{2}+k_{3}+k_{5} \\
& q=\left[p^{2}-4\left(k_{1} k_{3}+k_{2} k_{5}+k_{1} k_{5}\right)\right]^{1 / 3}
\end{aligned}
$$

Now $\quad \lim _{t \rightarrow \infty} \Delta_{r}=0, \quad r=1,2,3$.

Hence $\quad b_{r 1}=0, \quad r=1,2,3$.

The second term in the expression for $\Delta_{1}$, i.e. $b_{r 2} e^{-\lambda_{2} t}$, becomes negligible very quickly since $\lambda_{2}$ is large $\left.\left(k_{3}, k_{5}\right\rangle>k_{1}, k_{2}\right)$. Thus the relaxation is approximately described by

$$
\Delta_{r}=b_{r 3} e^{-\lambda_{2} t}
$$

and we have for the relaxation time

$$
\tau=1 / \lambda_{3}
$$

or, since $\left(k_{1} k_{3}+k_{2} k_{5}+k_{1} k_{5}\right)<<p^{2}$, we may approximate

$$
\begin{gathered}
\frac{1}{\tau}=\lambda_{3}=\frac{1}{2} p\left(1-\sqrt{1-4 \cdot \frac{k_{1} k_{3}+k_{2} k_{5}+k_{1} k_{5}}{p^{2}}}\right) \approx \\
\approx \frac{k_{1} k_{3}+k_{2} k_{5}+k_{1} k_{5}}{p}=\frac{k_{1} k_{3}+k_{2} k_{5}+k_{1} k_{5}}{k_{1}+k_{2}+k_{3}+k_{5}}
\end{gathered}
$$

Utilizing the fact that $k_{1}, k_{2}<<k_{3}, k_{5}$, we may approximate further

Resubstituting

$$
\frac{1}{\tau} \approx \frac{k_{1} k_{3}+k_{2} k_{5}+k_{1} k_{5}}{k_{3}+k_{5}}=k_{1}+\frac{k_{2} k_{5}}{k_{3}+k_{5}}
$$

we obtain

$$
k_{5}=2 k_{4} c_{ \pm}^{\circ}
$$

$$
\frac{1}{\tau} \approx k_{1}+\frac{2 k_{4} c_{ \pm}^{\circ} k_{2}}{2 k_{4} \mathrm{c}_{ \pm}^{\circ}+k_{3}}
$$

Denoting the true dissociation constant of $\mathrm{H}_{2} \mathrm{CO}_{3}$ by $K_{\mathrm{s}}=k_{3} / k_{4}$, we finally obtain

$$
\frac{1}{\tau} \approx k_{1}+\frac{2 c_{ \pm}^{\circ} k_{2}}{2 c_{ \pm}^{\circ}+K_{\mathrm{s}}}
$$

This expression agrees asymptotically with those given by Eigen ${ }^{24}$ for the two limiting cases $*, 2 c_{ \pm}^{\circ} \gg K_{s}$ and $\left.2 c_{ \pm}^{\circ}\right\rangle K_{s}$. The former is equivalent to the simple case treated in the preceding section.

* $2 c_{ \pm}^{\circ}<<K_{s}$ means that the dissociation of $\mathrm{H}_{2} \mathrm{CO}_{3}$ is practically complete, $i$. e. we are concerned with the reaction: $\mathrm{CO}_{2}+\mathrm{H}_{2} \mathrm{O} \rightleftharpoons \mathrm{H}++\mathrm{HCO}_{3}^{-}$. In this case we have

$$
1 / \tau=k_{1}+2 k_{2} c_{ \pm} / K_{s} \text {. }
$$

$2 c_{ \pm}^{\circ} \gg K_{s}$ means that the dissociation of $\mathrm{H}_{2} \mathrm{CO}_{3}$ is very small, $i$. $e$. we would have the reaction $\mathrm{CO}_{2}+\mathrm{H}_{2} \mathrm{O} \rightleftharpoons \mathrm{H}_{2} \mathrm{CO}_{3}$, with the rate of relaxation determined by $1 / \tau=k_{1}+k_{2}$.

Acta Chem. Scand. 12 (1958) No. 9 
b. Experimental. In view of the theoretical considerations given above, it is now possible by carrying out experiments at various concentrations to determine the rate constants $k_{1}, k_{2}$ and the true dissociation constant $K_{s}$ as well as the hydration constant $K_{h}$. For, utilizing the fact known from earlier investigations that $k_{1}<<k_{2} *$, the equation

$$
\frac{1}{\tau}=k_{1}+\frac{2 c_{ \pm}^{\circ} k_{2}}{2 c_{ \pm}^{\circ}+K_{\mathrm{s}}}
$$

may in our case be replaced by

$$
\tau=\frac{1}{k_{2}}\left(1+\frac{K_{s}}{2 c_{ \pm}^{\circ}}\right)
$$

Denoting the equivalent ionic conductances by $l$, we have for the conductivity (neglecting the difference between the actual equivalent conductivities and those at infinite dilution):

Now

$$
x=10^{-3} c_{ \pm}^{\circ}\left(l_{\mathrm{H}+}^{\circ}+l_{\mathrm{HCO}}^{\circ-\cdots}\right)
$$

$$
J=R \varkappa
$$

where $J$ is the cell constant and $R$ the resistance of the cell. Hence

$$
1 / c_{ \pm}^{\circ}=10^{-3}\left(l_{ \pm+}^{\circ}+l_{\mathrm{HCO}}^{\circ}\right) R / J
$$

If this is inserted in the expression for $\tau$ we obtain

$$
\tau=1 / k_{2}+10^{-3} K_{s}\left(l_{\mathrm{H}+}^{\circ}+l_{\mathrm{HCO}_{3}^{-}}^{\circ} R / 2 J k_{2}\right.
$$

Consequently we may plot $\tau$ versus $R$ to obtain a straight line.'

The results of the experiments are given in Table 1 and shown in Fig. 7.

Table 1. Chemical relaxation time for aqueous solutions ** of $\mathrm{CO}_{2}$ at $4^{\circ} \mathrm{C}$ and 150 atm pressure.

\begin{tabular}{ccc}
$R$ & $R_{\text {corr }} * * *$ & $\tau$ \\
\hline $5.24 k \Omega$ & $5.20 k \Omega$ & 0.76 sec \\
5.24 & 5.20 & 0.77 \\
5.98 & 5.93 & 0.62 \\
5.98 & 5.93 & 0.61 \\
9.13 & 9.06 & 0.75 \\
9.13 & 9.06 & 0.72 \\
9.13 & 9.06 & 0.83 \\
13.7 & 13.6 & 0.92 \\
13.7 & 13.6 & 0.95 \\
16.4 & 16.2 & 1.30 \\
16.4 & 16.2 & 1.19
\end{tabular}

* cf. Table 2.

** The carbon dioxide solutions were prepared by bubbling $\mathrm{CO}_{2}$ through conductivity water and then diluting to the desired concentration. Since losses of $\mathrm{CO}_{2}$ were difficult to avoid, it is more reliable to use the conductivity for the concentration determination throughout.

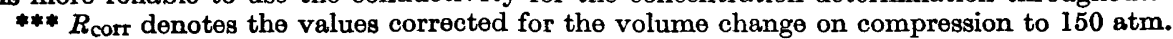




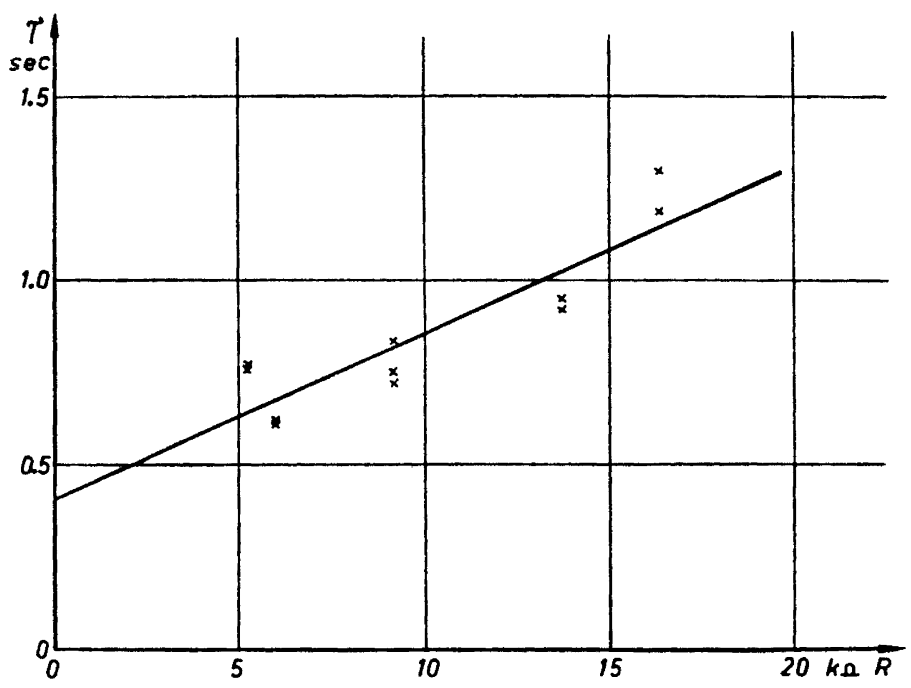

Fig. 7. Plot of $\tau$ versus $R$ from experiments with $\mathrm{CO}_{2}-\mathrm{H}_{2} \mathrm{O}$ at $4^{\circ} \mathrm{C}$.

A straight line has been fitted to the points in Fig. 7 using the method of least squares. The equation of this line is

where

$$
\tau=a R+b
$$

Thus

$$
\begin{aligned}
& a=(0.045 \pm 0.006) 10^{-3} \mathrm{sec} \mathrm{ohm}^{-1} \\
& b=(0.409 \pm 0.025) \mathrm{sec}
\end{aligned}
$$

and with

$$
k_{2}=1 / b=2.4 \mathrm{sec}^{-1}
$$

$$
K_{s}=2 \times 10^{3} J k_{2} a /\left(l_{\mathrm{H}+}^{\circ}+l_{\mathrm{HCO}}^{\circ}\right)
$$

insertion of the various quantities yields

$$
K_{s}=1.6 \times 10^{-4}
$$

In these experiments, a cell with $J=0.21 \mathrm{~cm}^{-1}$ was used.

If we denote the hydration constant of carbon dioxide by $K_{h}$ :

$$
K_{h}=c_{\mathrm{H}_{2} \mathrm{CO}_{\mathrm{s}}} / c_{\mathrm{CO}_{\mathrm{s}}}=k_{1} / k_{2}
$$

and the apparent primary dissociation constant of carbonic acid by $K_{s h}$ :

$$
K_{s h}=\frac{c_{\mathrm{H}+} \cdot c_{\mathrm{HCO}_{\mathrm{s}}}}{c_{\mathrm{CO}_{\mathrm{s}}}+c_{\mathrm{H}_{3} \mathrm{CO}_{3}}}
$$

Acta Chem. Scand. 12 (1958) No. 9 
it is well known that

$$
K_{s h}=\frac{K_{s} K_{h}}{1+K_{h}}
$$

Inserting our values for $K_{s}$ and $k_{2}$ and the known ${ }^{25}$ value of $K_{s h}$ (interpolation yields $K_{s h} \approx 2.94 \times 10^{-7}$ at $4^{\circ} \mathrm{C}$ ) we obtain

$$
\begin{aligned}
& K_{h}=1.8 \times 10^{-3} \\
& k_{1}=4.4 \times 10^{-3} \mathrm{sec}^{-1} \text { mole }^{-1} 1
\end{aligned}
$$

c. Discussion. The results of our present investigation at $4^{\circ} \mathrm{C}$ and 150 atm are *

$$
\begin{aligned}
& k_{1}=4.4 \times 10^{-3} \mathrm{sec}^{-1} \mathrm{~mole}^{-1} 1 \\
& k_{2}=2.4 \mathrm{sec}^{-1} \\
& K_{h}=1.8 \times 10^{-3} \\
& K_{s}=1.6 \times 10^{-4}
\end{aligned}
$$

Some previous results are given in Table 2 for comparison.

Table 2. Previous results for $k_{1}, k_{2}, K_{\mathrm{s}}$ and $K_{h}$.

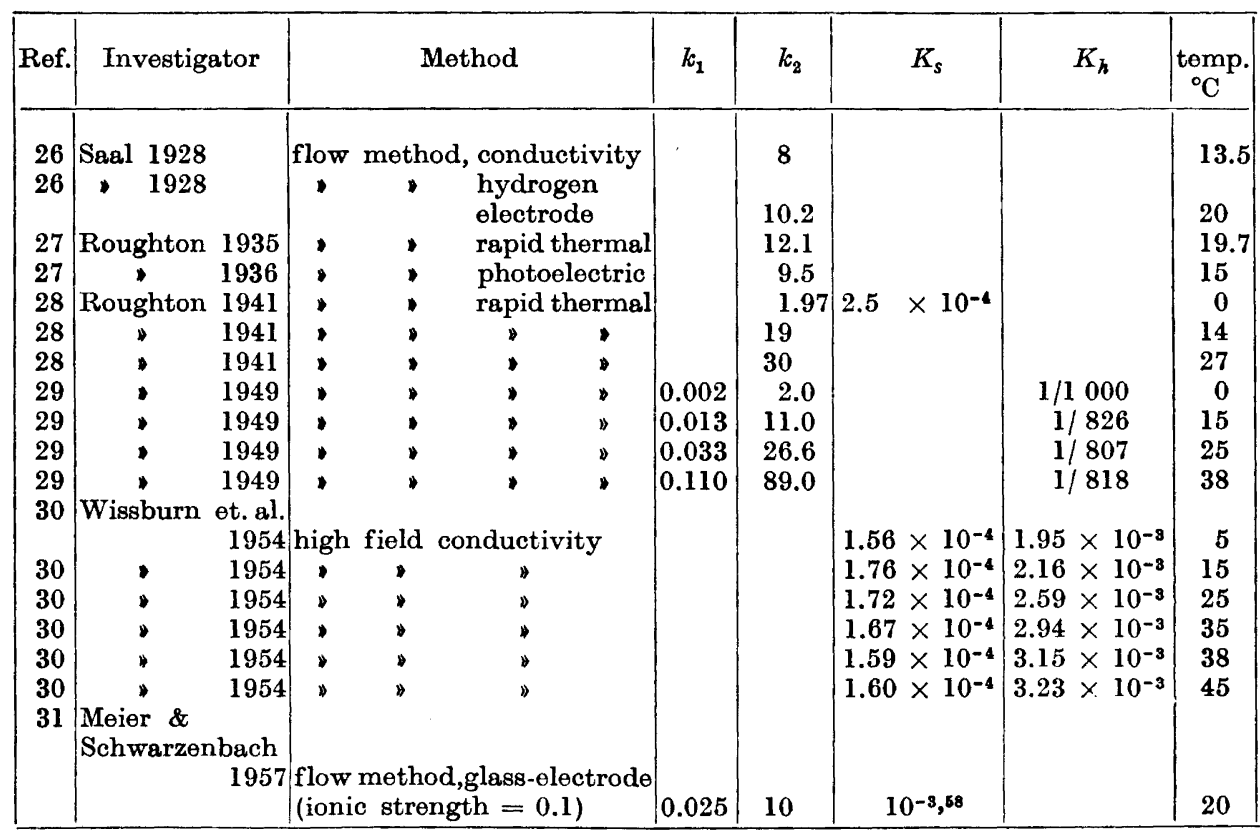

* It was observed that curves corresponding to a much shorter relaxation time were obtained at $18^{\circ} \mathrm{C}$ consistent with the temperature dependence of the rate constants. However, no quantitative experiments were carried out at this temperature.

By carrying out experiments at two different temperatures, it would, however, be possible to determine the activation energies of the various steps of the reaction between $\mathrm{CO}_{2}$ and water. 
The dispersion of the points in Fig. 7 indicates that the precision of the method is not yet very great. There are two imperfections in the electric circuit which should be mentioned. One is the deviation from linearity of the rectifier. Although the recording system was calibrated before each run, a certain loss of accuracy is always obtained. The other is the "overshoot" effect produced by the resonance compensating arrangement of the recorder. This imperfection is present in all mechanical oscillographs and cannot be avoided unless very particular measures are taken. These systematic erros together with the accidental errors of the recording system and errors in the evaluation of the relaxation curves cause an error in the $\tau$ values obtained of an order of magnitude from $10 \%$ to $20 \%$ in unfavourable cases. It seems however that, using more elaborate components in the electric system, it will be possible to increase the accuracy.

The low ionic concentrations in the experiments with the volatile $\mathrm{CO}_{2}$ water system makes these determinations particularly difficult.

In the experiments it was observed that the balancing capacitance of the cell had to be changed when the pressure was applied. This was considered to be without influence on the determinations. It would be possible, by the use of a phase sensitive rectifier, to study this effect which is, at least in part, of a purely mechanical nature (a change in the capacitance of the leads).

Acknowledgement. A pressure method for the determination of micelle formation rates was independently proposed several years ago by G. Aniansson of this Institute.

The financial support of Statens Naturvetenskapliga Forskningsråd is most gratefully acknowledged.

\section{REFERENCES}

1. Discussions Faraday Soc. 17 (1954) 114 f.f.

2. Weissberger, A. Technique of Organic Chemistry, vol. VIII, Ch. X, New York 1953.

2a. DuBois, D. J. Biol. Chem. 137 (1941) 123.

3. Onsager, L. J. Chem. Phys. 2 (1934) 599.

4. Harned, H. S. and Owen, B. B. The physical chemistry of electrolytic solutions, 3rd ed., New York 1958, p. 96.

5. Discussions Faraday Soc. 17 (1954) 202.

6. La Mer, V. K. and Read, C. L. J.Am. Chem. Soc. 52 (1930) 3098.

7. Loewenstein, A. J. Chem. Phys. 27 (1957) 1067.

8. Gilkersson, W. R. J. Chem. Phys. 27 (1957) 914.

9. Delahay, P. J. Am. Chem. Soc. 73 (1951) 4944.

10. Henke, K. H. and Hans, W. Z. Elektrochem. 57 (1953) 591, 595.

11. Vetter, K. J. Z. physik. Chem. 194 (1950) 199, 284.

12. Gerischer, H. and Vetter, K. J. Z. physik. Chem. 197 (1951) 92.

13. Gierst, L. and Juliard, A. J. Phys. Chem. 57 (1953) 701.

14. Delahay, P. and Berzius, T. J. Am. Chem. Soc. 75 (1953) 2486, 4205.

15. Gerischer, H. Z. physik. Chem. 2 (1/2) (1954) 79.

16. Hamman, S. D., and Linn, S. C. Australian J. Chem. 7 (1954) 329.

17. Bridgman, P. W. The physics of high pressure, London 1949.

18. Glasstone, S. Laidler, K. and Eyring, H. The theory of rate processes, Now York 1941, p. 470 .

18a. Van Santen, G. W. Introduction to a Study of Mechanical Vibration, 2nd ed., Eindhoven 1958, p. 52.

19. Carrington, T. and Davidson, N. J. Phys. Chem. 57 (1953) 418.

20. Discussions Faraday Soc. 17 (1954) 58.

21. Pinsent, B. and Roughton, F. Trans. Faraday Soc. 47 (1951) 263.

Acta Chem. Scand. 12 (1958) No. 9 
22. Frost, A. A. and Pearson, R. G. Kinetics and mechanism, New York 1953.

23. Matsen and Franklin J. Am. Chem. Soc. 72 (1950) 3337.

24. Eigen, M. Z. physik. Chem. 1 (3/4) (1954) 176.

25. Shedlovsky, T. and Mc Innes, D. J. Am. Chem. Soc. 57 (1935) 1705.

26. Saal Rec. trav. chim. 47 (1928) 264.

27. Roughton, F. and Millikan Proc. Roy. Soc. London A 155 (1936) 272.

28. Roughton, F. J. Am. Chem. Soc. 63 (1941) 2930.

29. Pinsent, B. and Roughton, F. Intern. Congr. Biochem., Abstrs of Communs, lst Congr., Cambridge, England, 1949, p. 375.

30. Wissburn, K. F. et al. J. Phys. Chem. 58 (1954) 693.

31. Meier, J. and Schwarzenbach, G. Helv. Chim. Acta 40 (4) (1957) 907.

Received July 9, 1958. 